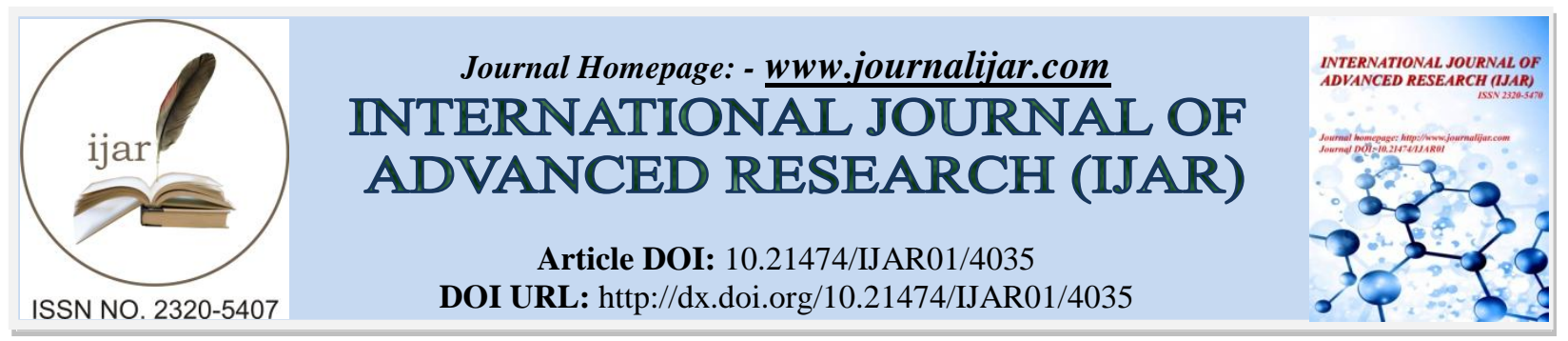

RESEARCH ARTICLE

\title{
SUPERLUMINAL VELOCITY OF ELECTROMAGNETIC WAVES IN PLASMA AND MONOCHROMATICITY.
}

Rakesh Kumar Pandey.

Kirori Mal College, University of Delhi, Delhi - 110007.

\section{Manuscript Info}

Manuscript History

Received: 28 February 2017

Final Accepted: 26 March 2017

Published: April 2017

Key words:-

Quantum Mechanics, Electromagnetic

wave, Plasma, Uncertainty relation,

Wave-particle duality, Theory of

Relativity

\begin{abstract}
It is shown that when the expression of velocity of electromagnetic waves in Plasma is derived using the Maxwell's relations its velocity turns out to be violating the ultimate limit on the velocities as it allows its velocity to be greater than the velocity of light in the vacuum. It is then shown that while only phase velocity of the electromagnetic wave threatens to cross the limit, the group velocity still respects the upper limit on the velocity and remains smaller than the velocity of light in vacuum. Based on this, it is argued that admissibility of the existence of a strictly monochromatic electromagnetic wave would violate the inherent wave-particle dual nature of the wave and hence is a rejected possibility.
\end{abstract}

Copy Right, IJAR, 2017,. All rights reserved.

\section{Introduction:-}

Propagation of electromagnetic waves in Plasma is a well understood phenomena ${ }^{2,6}$ but the strange fact is that when Maxwell's relations are applied in such a case it gives an expression for the velocity of electromagnetic waves that permits it to be more than the velocity of light in the vacuum. This places a challenge before us to have a complete understanding about the propagation. This paper shows that it is actually the phase velocity that crosses the limit of velocity of light in the vacuum whereas the group velocity still observes the same limit without any scope of violation.

Based on this it is argued that electromagnetic waves can never be strictly monochromatic in nature. This inherent nature asks all electromagnetic waves to be a group of waves wherein the waves must have a spread in their frequencies around a particular frequency even when a monochromatic wave is considered.

The first section shows how in the case of Plasma the Maxwell relations give an expression for the velocity of an electromagnetic wave that potentially violates the upper limit on the velocities as given by the Einstein's Theory of Relativity.

The later section explains how the concept of group velocity addresses this concern. It is shown that in case of Plasma the group velocity can never cross the Theory of Relativity limit on the velocities.

In the last section it is then argued that it is simply impossible to have a strictly monochromatic electromagnetic wave in principle as it will then violate the accepted limit on its velocity. Therefore it is suggested that in order to have the essential wave particle duality ${ }^{8,9}$ the electromagnetic waves can never be completely monochromatic. 


\section{Propagation of electromagnetic wave in Plasma:-}

Propagation of electromagnetic wave inside Plasma has remained of interest to physicists for a long time. Plasma is considered as an ensemble of non-colliding freely moving ions ${ }^{2,6}$ almost as a collection of slowly moving gas molecules. An electric field set up across such a medium drives these charges with a force given by,

(1)

$$
\vec{F}=\frac{d(m \vec{v})}{d t}=e \vec{E}
$$

Where $m$ and $v$ are the mass and drift velocity of these ions. We also know that the current density is related to the drift velocity as given by the relation,

$$
\vec{J}=n e \vec{v}
$$

where ' $n$ ' is the number of ions per unit volume of the Plasma medium and ' $e$ ' is the charge on these ions. Differentiating equation (2) gives,

$$
\frac{d \vec{J}}{d t}=n e \frac{d \vec{v}}{d t}
$$

Equation (1) then gives,

$$
\frac{d \vec{J}}{d t}=\left(n e^{2} \vec{E}\right) / m
$$

Using the standard procedure to find out the solutions in such a situation we replace $\frac{d}{d t}$ with $i \omega$ ( $\omega$ being the frequency of the electromagnetic wave and $i$ as square root of -1) and $\vec{\nabla}$ with $-i \vec{k}$ ( $\vec{k}$ being the propagation vector) we get a relation between the current density and the electric field as,

$$
\vec{J}=-i n \frac{e^{2}}{m \omega} \vec{E}
$$

Comparing this relation with the relation between $\vec{J}$ and $\vec{E}$ in case of conducting medium as $\vec{J}=\sigma \vec{E}$, Plasma can be considered as a 'conducting' medium having purely imaginary conductivity given by

(6)

$$
\sigma=i n \frac{e^{2}}{m \omega}
$$

Taking curl of third Maxwell's relation and approximating $\varepsilon$ and $\mu$ as $\varepsilon_{0} \& \mu_{0}$ respectively by assuming the medium as a non-conducting Plasma medium and also by considering free net charge to be zero we can arrive at the following relation for the propagation of electromagnetic waves in Plasma,

$$
\nabla^{2} \vec{E}=\mu_{0}\left(\frac{d \vec{J}}{d t}+\varepsilon_{0} \frac{d^{2} \vec{E}}{d t^{2}}\right)
$$

Replacing again $\frac{d}{d t}$ with $i \omega$ ( $\omega$ being the frequency of the electromagnetic wave and $i$ as square root of -1$)$ and $\vec{\nabla}$ with $-i \vec{k}$ ( $\vec{k}$ being the propagation vector) and thereafter simplifying the expression we can easily arrive at the following expression,

$$
k^{2}=\left(\omega^{2} / c^{2}\right)\left(1-\left(\omega_{p} / \omega\right)^{2}\right)
$$

Where $\omega_{p}$ is the plasma frequency given by

(9)

$$
\omega_{p}{ }^{2}=\left(n e^{2}\right) /\left(m \varepsilon_{0}\right)
$$

From the above relation, the velocity of wave that can be derived easily by determining the ratio $\omega / k$ and arrive at the following relation

$$
v=c\left(1-\left(\omega_{p} / \omega\right)^{2}\right)^{-1}
$$


It has been shown in the next section that $\omega_{p}$ gives the critical value of the frequency for a particular kind of Plasma that decides whether an electromagnetic wave would be able to travel inside it or not. This frequency $\omega_{p}$ is known as Plasma frequency.

\section{Velocity of electromagnetic wave crosses the limit of velocity of light in the vacuum:-}

Equation (8) shows that for frequencies of the waves which are less than $\omega_{p}$ the propagation constant will be purely imaginary leading to the conclusion that the amplitude of the wave will get attenuated exponentially and therefore the wave can not travel in the medium ${ }^{2,6}$. Skin depth in such a scenario can be given by the relation

$$
\delta=(\text { imaginary } k)^{-1}=\left((c / \omega)\left(\left(\frac{\omega_{p}}{\omega}\right)^{2}-1\right)^{-\frac{1}{2}}\right.
$$

On the other hand, only those waves that will have frequencies more than the Plasma frequency $\omega_{p}$ will be able to travel in the plasma as then the propagation constant will be real. Velocity of these waves will be given by the equation (10). Remarkably, it is this theory that explains why radio waves of smaller frequencies (AM) does not penetrate the Plasma like ionosphere and gets reflected back to the earth leading to a much greater coverage of these radio waves on earth ${ }^{6,7}$. Whereas FM radio waves of larger frequencies penetrates the ionosphere to move into the space and does not come back on the earth. Due to this well understood fact, AM radio waves covers a larger area on earth but FM waves can spread in an area that gets determined solely by the height of the tower. The area of coverage for such a wave increases with the height that can be estimated by pure geometry of the case.

Typically if one calculates the Plasma frequency of the Plasma like ionosphere by putting the respective values, it can be shown that it turns out to be around $3 \mathrm{MHz}^{6}$. It has now been practically verified that all electromagnetic waves with frequencies significantly larger than this penetrates the ionosphere and get lost in the outer space but waves having frequencies less than this indeed comes back after getting reflected by the ionosphere. This understanding is also utilized for securing communication from satellites for choosing the frequency of the wave in the communication.

Quiet strangely, although the relation (10) permits the velocities to cross the Relativity limit on the velocity, such a violation has never been observed or reported. And even the refractive index of such a medium that can be given by the expression

$$
\frac{c}{v}=1-\left(\omega_{p} / \omega\right)^{2}
$$

turns out to be less than one! This paper focuses only on the case when the wave penetrates inside the Plasma and propagates in the medium but yet never violates the velocity as derived by the equation (10).

The equation (10) clearly violates the Einstein's Theory of Relativity that demands an unassailable limit on all such velocities as the velocity of light in the vacuum. It can be shown that any such violation would break down our understanding of the universe. Our 'known' or 'observable' universe gets limited by the fact that we can never be able to get a wave that moves faster than light. And the moment we would accept its violation we would be able to 'see' the future through these waves which is simply impossible and unimaginable.

\section{The Explanation:-}

It is not that this can be reported as a unique case where the velocity of a wave threatens to cross the upper limit on the velocities as we have a few other cases too. Gunter Nimtz and Alfons A. Stahlhofen conducted an experiment ${ }^{4}$ and reported velocities greater than the velocity of light but not in the case of propagation in Plasma. Superluminal signals have been reported by them in the case when particles travel inside a forbidden potential barrier. This is widely reported and discussed and physicists are trying to address those issues also ${ }^{1,4,5}$.

This problem that we have identified in this paper however can be explained by the fact that light can never be strictly monochromatic in nature and will always be associated with a number of waves having their frequencies spread around the frequency of light that would allow them eventually to group themselves together to form a 'real' electromagnetic wave. Such a picture of the electromagnetic wave will explain the simultaneous particle nature of light as the wave-particle duality of photons is a well established fact. And this being the proposed picture, to estimate the velocity of wave we must determine velocity of the group of waves as given by $v_{g}=\frac{d \omega}{d k}$ and not by the phase velocity $v_{p}=\frac{\omega}{k}$. 
Let us determine the group velocity $v_{g}$ in this particular case. Group velocity can be derived by simply differentiating the equation (8) to get the following expression

$$
v_{g}=\frac{d \omega}{d k}=c\left(1-\left(\omega_{p} / \omega\right)^{2}\right)^{1 / 2}
$$

Clearly, as $\left(1-\left(\omega_{p} / \omega\right)^{2}\right)^{1 / 2}$ will always remain less than one for $\omega$ more than $\omega_{p}$ the group velocity will observe the ultimate limit on the velocity and $v_{g}$ will remain less than $\mathrm{c}$ as desired for consistency. This finally explains the propagation of electromagnetic waves in the Plasma when $\omega_{p}$ happens to be less than $\omega$.

\section{Discussion:-}

The above explanation gives an insight into our understanding of the electromagnetic waves. We must therefore, accept that electromagnetic waves can never exist as a strict monochromatic entity. There must be an inherent 'nonmonochromaticity' associated with the waves otherwise it would potentially violate the upper limit on the velocities. The manner in which quantum mechanics argues that monochromatic waves will simply fail to represent a particle ${ }^{8}$, we must extend the same logic to accept that a strictly monochromatic electromagnetic wave can not represent a photon (particle). It is this inherent dual property of matter-wave that defines the real world as per our understanding developed after Quantum Mechanics ${ }^{3,10}$. The Heisenberg's Uncertainty principle also finds consistency with only such a picture ${ }^{8,9}$.

One must notice that the Heisenberg Uncertainty Relation also can not accept the existence of a 'particle at rest' as this picture of the particle violates the very Principle. A 'particle at rest' clearly implies that in this picture 'position' and the 'momentum' of the particle appear to be precisely known!

This explains why electromagnetic waves can never have infinite 'coherence length' or an infinite 'coherence time' as this would require the wave to be of strictly monochromatic in nature. Therefore it is now clear that 'strict monochromaticity' is unphysical and would challenge the wave-particle duality.

Just as a 'particle at rest' picture depicts a unilateral 'particle picture' without a trace of its wave characteristics, the 'monochromaticity' demands a unilateral 'wave picture' of the physical entity to be accepted without any particle characteristics. However, if the wave-particle duality is an inherent property then the Uncertainty Principle would demand rejection of both these pictures that violates the essential requirement of duality.

\section{References:-}

1. Chiao, R. Y., Kwiat, P. G. and Steinberg A. M., Faster than light? Scientific American, 1993, pages 38 - 46

2. Jackson J D, 1989, "Classical Electrodynamics", Second Edition, Wiley Eastern Limited.

3. Nairz O, Arndt M, Zeilinger A, Experimental verification of the Heisenberg Uncertainty Principle for Fullerene molecules, Physical Review A 65(3), 2002: DOI: 10.1103/PHYSREVA.65.032109.

4. Nimtz G and Stahlhofen A A, Macroscopic violation of Special Theory of Relativity, arXiv August 2007, arXiv:0708.0681vl

5. Nimtz, G. and Heitmann W., Superluminal Photonic Tunneling and Quantum Electronics. Progress in Quantum Electronics,21, 81 (1997)

6. Paul Lorrain and Dale Corson, 1969, "Electromagnetic Fields and Waves", Second Edition, CBS Publishers \& Distributors

7. Puri S P, 1997, "Classical Electrodynamics", Second Edition, Tata McGraw-Hill Publishing Company Limited.

8. Robert Eisberg and Robert Resnick, 2004, "Quantum Physics of Atoms, Molecules, Solids, Nuclei and Particles", Second Edition, John Wiley \& Sons

9. Stephen Gasiorowics, 2000, "Quantum Physics", Second Edition, John Wiley \& Sons, Inc.

10. Thom JJ, Neel MS, Donato VW, Bergreen GS, Davies RE, Beck M, Observing the Quantum Behaviour of Light in an undergraduate Laboratory, American Journal of Physics 72(9), 1210-1219, 2004. 